\title{
F.F.T. Hashing is not Collision-free
}

\author{
T. BARITAUD * , H. GILBERT * , M. GIRAULT **
}

(*) CNET PAA / TSA / SRC

38 - 40, avenuc du Génćral Leclere

92131 ISSY LES MOULINEAUX (France)
(**) SEPT PEM

42, ruc des Coutures

BP 6243

14066 CAEN (Francc)

\section{Abstract}

The FFT Hashing Function proposed by C.P. Schnorr [1] hashes messages of arbitrary length into a 128bit hash value. In this paper, we show that this function is not collisian free, and we give an example of two distinct 256-bit messages with the same hash value. Finding a collision (in fact a large family of, colliding messages) requires approximately 23 partial computations of the hash function, and lakes a few hours on a SUN3-workstation. and less than an hour on a SPARC-workstation.

A similar result discovered independently has been announced at the Asiacrypt 91 rump session by Daemen-Bosselaers-Govaeris-Vandewalle 121.

\section{The FFT Hashing Function}

\subsection{The Hash algorithm}

Let the message be given as a bit string $m_{1} m_{2} \ldots m_{t}$ of $\mathrm{L}$ bit.

The message is first padded so that its length (in bits) becomes a multiple of 128. Let the padded messagc $M_{1} M_{2} \ldots M_{n}$ consist of $n$ blocks $M_{1}, \ldots, M_{n}$, cach of the $M_{i}(i=1, \ldots, n)$ being 128-bit long.

The algorithm uses a constant initial value $\mathrm{H}_{0}$ given in hexadecimal as

$$
\mathrm{H}_{0}=0123456789 \mathrm{ab} \text { cdef fedc ba98 } 76543210 \text { in }\{0,1\}^{128} \text {. }
$$


Let $p$ be the prime $65537=2^{16}+1$.

We will use the Fourier transform $\left.\mathrm{FT}_{8}:\{0, \ldots, \mathrm{p}-1\}^{8} \ldots \ldots, \ldots, \mathrm{p}-1\right\}^{8}$

$$
\left(a_{0}, \ldots, a_{7}\right) \cdots \cdots\left(b_{0}, \ldots, b_{7}\right)
$$

with $b_{i}=\sum_{j=0}^{7} 2^{4 i j} a_{j} \bmod p$, for $i=0, \ldots, 7$.

Algorithm for the hash function $\mathrm{h}$ :

$$
\begin{aligned}
& \text { INPUT : } M_{1} M_{2} \ldots M_{n} \text { in }(0,1)^{n .128} \text { (a padded mcssagc) } \\
& \text { DO : } H_{i}=g\left(H_{i-1}, M_{i}\right) \quad \text { lor } i=1, \ldots, n \\
& \text { OUTPUT: } \quad h(M):=H_{n}
\end{aligned}
$$

Algorithm for $g: z_{p}^{16} \ldots \ldots,[0,1]^{8.16}$

$$
\begin{array}{ll}
\text { INPUT } & \left.\left(\mathrm{c}_{0}, \ldots, c_{15}\right) \text { in }\{0,1\}\right)^{16.16} \\
\text { 1. } & \left(\mathrm{c}_{0}, \mathrm{c}_{2}, \ldots, \mathrm{e}_{14}\right):=\mathrm{FT}_{8}\left(\mathrm{c}_{0}, \mathrm{c}_{2}, \ldots, \mathrm{c}_{14}\right) \\
\text { 2. } & \text { FOR } \mathrm{i}=0, \ldots, 15 \mathrm{DO} \\
& \mathrm{e}_{\mathrm{i}}:=\mathrm{c}_{\mathrm{i}}+\mathrm{c}_{\mathrm{i}-1} \mathrm{e}_{\mathrm{i}-2}+\mathrm{c}_{\mathrm{c}_{\mathrm{i}-3}}+2^{\mathrm{i}}(\bmod \mathrm{p})
\end{array}
$$

(The lower indices $i, i-1, i-2, i-3, c_{i-3}$ are aken modulo 16)

3. REPEAT sleps 1 and 2

$$
\text { OUTPUT } \quad \bar{c}_{i}:=c_{i} \bmod 2^{16}, \text { for } i=8, \ldots, 15 \text { (an clement of }[0,1\}^{8.16} \text { ) }
$$

\subsection{Notations}

For a beller clarity of our explanation, we will denote by $c_{i}^{0}(i=0, \ldots, 15)$ the initial $c_{i}$ values, and we will denote by step 3 (resp. step 4) the second pass of step 1 (resp. step2) in the algorithm for g.

When it will be necessary to avoid any kind of slip, we will denote by $c_{i}^{k}(i=0, \ldots, 15 ; k=0, \ldots, 4)$ the $c_{i}$ intermediate value, after step $k$. 
In order to simplify the expressions, we are using the following notations :

- The additions $(x+y)$, multiplications $(x . y)$ and exponcntiations $\left(x^{y}\right)$ are implicitly made modulo p, except when the operands are lower indices.

- The = symbol denotes that the right and the left terms are congruent modulo $p$.

- For lower indices the additions $(i+j)$ and substractions $(i-j)$ are implicilly made modulo 16 , and the $\equiv$ symbol denotes that the right and the left terms are congruent modulo 16.

\subsection{Preliminary remarks}

The difficulty of finding collisions is related to the diffusion properties of the hashing function, i.c. the influence of a modification of an intermediate variable on the subscquent variables of the calculation.

\section{Remark 1 (limitation on the diffusion at steps 1 and 3)}

At stcp 1 and 3, the inpul values $c_{1}, c_{2}, \ldots, c_{15}$ arc kcpt unchanged.

\section{Remark 2 (limitation on the diffusion at steps 2 and 4)}

The diffusion introduced by the $c_{\mathrm{i}-1} \mathrm{c}_{\mathrm{i}-2}$ terms in the recurrence for steps 2 and 4 can sometimes be cancelled (if one of values $e_{i-1}$ and $c_{i-2}$ is 0 ). More preciscly, let $\left(c_{0}^{1}, c_{1}^{1}, \ldots, e_{15}^{1}\right)$ be the input to step 2 :

Proposition 1 : If for a given value $i$ in $(1, \ldots, 14\}$ we have $c_{i-1}^{2}=c_{i+1}^{2}=0$ and ir $c_{13}^{1} \equiv i ; c_{14}^{1} \neq i$ $e_{15}^{1} \equiv i ; c_{j}^{2} \equiv i$ for $j$ in $[0, \ldots, 12]$, then the impact of replacing the input value $c_{i}^{1}$ by a new valuc $e_{i}^{1}+\Delta e_{i}^{1}$ such that $c_{i}^{1}+\Delta c_{i}^{1} \equiv c_{i}^{1}$, is linited to the output value $c_{i}^{2}$ (that means $c_{j}^{2}$ are not modificu for $j \neq i$ ).

Proposition 2: If $\mathrm{e}_{14}^{1}=\mathrm{e}_{0}^{2}=0$ and if $\mathrm{e}_{\mathrm{j}}^{2} \equiv 15$ for $\mathrm{j}$ in $(1, \ldots, 11)$ then the impact of replacing the input value $e_{15}^{1}$ by a new value $e_{15}^{1}+\Delta c_{15}^{1}$ such that $e_{15}^{1}+\Delta e_{15}^{1} \equiv c_{15}^{1}$, is limited to the output value $c_{15}^{2}$.

Similarly, let $\left(c_{1}^{3}, c_{2}^{3}, \ldots, c_{15}^{3}\right)$ be the input to step 4 :

Propesilion $\mathrm{l}^{\prime}$ : If for a given value $i$ in $\{1, \ldots, 14\}$ we have $e_{i-1}^{4}=e_{i+1}^{4}=0$ and if $e_{13}^{3} \neq i$; $c_{14}^{3} \equiv \mathrm{i}: c_{15}^{3} \equiv \mathrm{i} ; \mathrm{c}_{\mathrm{j}}^{4} \neq \mathrm{i}$ for $\mathrm{j}$ in $\{0, \ldots, 12\}$, then the impact of replacing the input value $\mathrm{c}_{\mathrm{i}}^{3}$ by a new valuc $e_{i}^{3}+\Delta c_{i}^{3}$ such that $c_{i}^{3}+\Delta c_{i}^{3} \equiv e_{j}^{3}$, is limitcd to the output value $c_{i}^{4}$. 
Proposition 2': If $c_{14}^{3}=e_{0}^{4}=0$ and if $c_{j}^{4} \neq 15$ for $j$ in $\{1, \ldots, 11\}$ then the impact of replacing the input value $e_{15}^{3}$ by a ncw value $e_{15}^{3}+\Delta c_{15}^{3}$ such that $e_{15}^{3}+\Delta c_{15}^{3} \equiv c_{15}^{3}$ is limited to the output value $e_{15}^{4}$.

\section{Construction of two colliding messages}

\subsection{Construction of a partial collision}

We first find two 128-bit blocks $M_{1}$ and $M_{1}^{\prime}$ which hash values $H_{1}=\left(\bar{c}_{8}^{4}, \ldots, \bar{e} \frac{4}{15}\right)$ and $\mathrm{H}_{1}^{\prime}=\left({\overline{\mathrm{c}^{\prime}}}_{8}^{4}, \ldots, \overline{\mathrm{c}^{\prime}}{ }_{15}^{4}\right)$ differ only by their right components $\overline{\mathrm{c}}_{15}^{4}$ and $\overline{\mathrm{c}}_{15}^{4}$. We will later refer to this property in saying that $M_{1}$ and $M_{1}^{\prime}$ realize a partialcollision.

Our technique for finding $M_{1}$ and $M_{1}^{+}$is the following : we search $M_{1}$ values such that $c_{14}^{1}=0$; $c_{0}^{2}=0 ; e_{14}^{3}=0 ; c_{0}^{4}=0$. The propositions 2 and 2 suggest that for such a messagc $M_{1}=\left(c_{8}^{0} \ldots, c_{14}^{0}, e_{15}^{0}\right)$, $M_{1}$ and the message $M_{1}^{\prime}=\left(e_{8}^{0}, \ldots, e_{14}^{0}, c_{15}^{0}+16\right)$ ralize a partial collision with a significant probability (approximatcly $1 / 8$ ).

There are two main steps for finding $M_{1}$.

Slepl : Sclection of $\mathrm{e}_{8}^{0}, \mathrm{e}_{10}^{0}, \mathrm{e}_{12}^{0}$ and $\mathrm{e}_{14}^{0}$

Arbitrary (c.g. random) values are taken for $\mathrm{e}_{12}^{0}$ and $\mathrm{c}_{14}^{0}$. The values of $\mathrm{e}_{8}^{0}$ and $\mathrm{c}_{10}^{0}$ are then deduced from these values by solving the following linear system:

$$
\left\{\begin{array}{l}
e_{14}^{1}=0 \\
e_{0}^{1}=-1
\end{array}\right.
$$

\section{Proposition 3:}

If $e_{13}^{0} \equiv 14$ then $c_{14}^{1}=0$ and $c_{0}^{2}=0$ independenlly of the values of $c_{9}^{0}, e_{11}^{0}, e_{13}^{0}, e_{15}^{0}$.

Broof: This is a direct consequence of the definition of the g function. 
SLep 2: Sclection of $c_{9}^{0}, e_{11}^{0}, c_{13}^{0}, c_{15}^{0}$

The values of $\mathrm{e}_{8,}^{0} \mathrm{e}_{10}^{0}, \mathrm{e}_{12}^{0}, \mathrm{e}_{14}^{0}$ are taken from Sicp 1 .

We fix the values of $c_{11}^{0}=0$ and $e_{15}^{0}=0$. An arbitrary (c.g random) value is taken for $e_{9}^{0}$ - We first calculate the $\mathrm{c}_{12}^{2}$ and $\mathrm{c}_{14}^{3}$ values corresponding to the chosen value of $c_{9}^{0}, c_{11}^{0}$ and $c_{15}^{0}$ and to the temporary value $\mathrm{c}_{13}^{0}=14$. Based on these preliminary calculations, we "correct" the temporary value $c_{13}^{0}=14$ by a quantity $\Delta c_{13}^{0}$, i.c. we replace the value $c_{13}^{0}=14$ by the value $c_{13}^{0}=14+\Delta c_{13}^{0}$, and we leave the other input values unchanged. We denote by $\Delta c_{j}^{i}(0 \leq i \leq 4 ; 0 \leq j \leq 15)$ the corresponding variations of the intermediate variabics in the $H_{1}$ calculation. We select $\Delta c_{13}^{0}$ in such a way that the quantity $c_{14}^{3}+\Delta c_{14}^{3}$ (i.e the new valuc of $\mathrm{c}_{14}^{3}$ ) is cqual to zero with a good probability.

Proposition 4 : If $\mathrm{c}_{12}^{2} \neq 0$ and $\frac{-\mathrm{e}_{14}^{3}}{2^{4.7 .7} \mathrm{e}_{12}^{2}} \equiv 0$ and $\mathrm{e}_{\mathrm{j}}^{2} \neq 13$ for $1 \leq \mathrm{j} \leq 11$ then the above values of $, c_{15}^{1}, c_{0}^{2}$ and the value $\Delta c_{13}^{0}=\frac{-c_{14}^{3}}{2^{4.7 .7} c_{12}^{2}}$ lead to the threc relations

$$
\left\{\begin{array}{l}
c_{14}^{1}+\Delta c_{14}^{1}=0 \\
c_{0}^{2}+\Delta c_{0}^{2}=0 \\
c_{14}^{3}+\Delta c_{14}^{3}=0
\end{array}\right.
$$

Broof : (a) is straightforward; (b) and (c) are direct consequences of the following relations, which result from the definition of the $\mathrm{g}$ function :

$$
\Delta c_{j-2}^{2}=0 \text { for } 0 \leq j \leq 12 ; \Delta c_{13}^{2}=\Delta c_{13}^{0} ; \Delta c_{14}^{2}=c_{12}^{2} \cdot \Delta c_{13}^{2} ; \Delta c_{14}^{3}=2^{4.7 .7} \cdot \Delta c_{14}^{2}
$$

We performed a large number $n_{1}$ of trials of step 1. For cach trial of step 1, we made a large number $n_{2}$ of trials of step 2 . The success probability of $\sec 2$, i.c the probability that the trial of a $c_{9}^{0}$ valuc leads to a message such that (a). (b) and (c) are realized is slightly less than $1 / 16$ (since the strongest 
condition in proposition 2 is : $\frac{-c_{14}^{3}}{2.4 .72} c_{12}^{3} \equiv 0$ ). Therefore the probability that a step 2 trial leads to a messagc $M_{1}$ such that $c_{14}^{1}=c_{0}^{2}=c_{14}^{3}=c_{0}^{4}=0$ is slightly less than $1 / 16.2^{-16}=2^{-20}$.

Morcover, the probability that such a message $M_{1}$ leads to a partial collision is basically the probability that none of the $e_{i-3} \bmod 16$ indices occurring in the calculation of $c_{0}^{2}$ to $c_{15}^{2}$ and $c_{0}^{4}$ to $c_{15}^{4}$ takes the value 15 , which is close to $1 / 8$. So, in summary, approximativcly $2^{23}$ partial computations of the $\mathrm{g}$ function were necessary to obtain a suitable message $M_{1}=\left(c_{8}^{0}, \ldots, c_{14}^{0}, c_{15}^{0}\right)$, such that $M_{1}$ and the message $M_{1}^{\prime}=\left(c_{8}^{0}, \ldots, c_{14}^{0}, c_{15}^{0}+16\right)$ icad to partially colliding hash valucs $H_{1}=\left(\bar{c}_{8}^{4}, \ldots, c_{15}^{4}\right)$ and $H_{1}=\left(\bar{c}_{8}^{-4}, \ldots, \bar{c}_{15}^{-4}+16\right)$

\subsection{Construction of a full collision using a partial collision}

We now show how to find a 128-bit message $M_{2}=\left(c_{8}^{0}, \ldots, c_{15}^{0}\right)$ such that the previously oblaincd

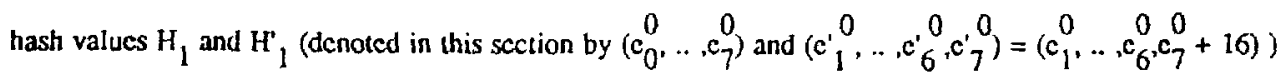
respectively lead to the same hash value $\mathrm{H}_{2}$ (when combincd with $\left.\mathrm{M}_{2}\right): g\left(\mathrm{H}_{1}, \mathrm{M}_{2}\right)=\mathrm{g}\left(\mathrm{H}_{1}, \mathrm{M}_{2}\right)$.

Our technique for finding $\mathrm{M}_{2}$ is quite similar to the one used for finding $M_{1}$ and $M_{1}^{\prime}$. Let us denote by $\mathrm{c}_{\mathrm{j}}^{\mathrm{i}}$ (resp $\left.\mathrm{c}_{\mathrm{j}}^{\mathrm{i}}\right)(0 \leq \mathrm{i} \leq 4,0 \leq j \leq 15)$ the intermediate variables of the calculations of $\mathrm{g}\left(\mathrm{H}_{1}, \mathrm{M}_{2}\right)$ (resp $\left.\mathrm{g}\left(\mathrm{H}_{1}, \mathrm{M}_{2}\right)\right)$

We scarch $M_{2}$ valucs such that $c_{6}^{2}=c_{8}^{2}=c_{6}^{4}=c_{8}^{4}=0$. The propositions 1 and 1 ' suggest that the probability that the 16-uples $\left(c_{0}^{4}, \ldots, e_{15}^{4}\right)$ and $\left(c_{0}^{\prime 4}, \ldots, e_{15}^{4}\right)$ differ only by their components $c_{7}^{4}$ and $e_{7}^{4}$ which implies that the probability to have $\mathrm{g}\left(\mathrm{H}_{1}, \mathrm{M}_{2}\right)=\mathrm{g}\left(\mathrm{H}_{1}, \mathrm{M}_{2}\right)$ is quitc substantial, approximatively $1 / 8$. 
There are two main steps for the search of $\mathrm{M}_{2}$ :

Sice 1: Sclection of $c_{8}^{0}, c_{10}^{0}, c_{12}^{0}, c_{14}^{0}, c_{9}^{0}$

An arbitrary (c.g random) value is taken for $c_{14}^{0}$. The values of $c_{8}^{0}, c_{10}^{0}, c_{12}^{0}$ are deduced from $c_{14}^{0}$ by solving the following lincar system :

$$
\left\{\begin{array}{l}
c_{14}^{1}=0 \\
c_{0}^{1}=-1 \\
c_{8}^{1}=-2^{8}
\end{array}\right.
$$

A preliminary calculation, where $e_{9}^{0}, c_{11}^{0}$ and $e_{15}^{0}$ are set to the temporary value 0 and $\mathrm{e}_{13}^{0}$ is set to the tcmporary value 14 , is made. The obtaincd valuc of $\mathrm{c}_{6}^{2}$, denoted by $\delta$, is kept.

Proposition 5 : If $\quad c_{8}^{0}, c_{10}^{0}, c_{12}^{0}, c_{14}^{0}$ are solutions of (3), (4), (5) and if in addition the values $e_{9}^{0}=p-\delta, c_{11}^{0}=0, c_{13}^{0}=14, e_{15}^{0}=0$ lcad to intermediate valucs such that : $e_{1}^{2}$ mod 16 is not in $\{9,11,13,15) ; \quad c_{2}^{2} \bmod 16$ is $\operatorname{not}$ in $(9,11,13,15\} ; \quad c_{3}^{2} \equiv 9 \bmod 16 ; \quad e_{4}^{2} \bmod 16$ is not in $(9,11,13,15\}$; $\mathrm{c}_{5}^{2} \bmod 16$ is in $(0,6,14)$, then if we fix the value $\mathrm{c}_{9}^{0}=p-\delta$, for any value of $c_{13}^{0} \equiv 14$ and for any value of $\mathrm{e}_{15}^{0}$ such that $\mathrm{c}_{15}^{0} \equiv 0$ we have :

$$
\mathrm{e}_{14}^{1}=0 ; \quad \mathrm{e}_{0}^{2}=0 ; \quad \mathrm{c}_{6}^{2}=0 ; \quad \mathrm{e}_{8}^{2}=0
$$

Proof: The proof of this proposition is casy. Finding the $c_{8}^{0}, c_{10}^{0}, c_{12}^{0}, c_{14}^{0}$ and $c_{9}^{0}$ values satisfying the conditions of the above proposition is quite casy, and requires the urial of a few hundreds $c_{14}^{0}$ valucs.

Slep 2: Sclcction of $c_{11}^{0}, c_{13}^{0}, c_{15}^{0}$

The values of $c_{8}^{0}, c_{10}^{0}, c_{12}^{0}, e_{14}^{0}, c_{9}^{0}$ are taken from Sicp 1; these values are assumed to realize the conditions of the above proposition.

An arbitrary (c.g random) value is taken for $c_{11}^{0}$. A preliminary calculation is made, using the sclected $c_{11}^{0}$ value and the temporary values $c_{13}^{0}=14 ; c_{15}^{0}=0$. The corresponding values of $c_{12}^{2}$ and $c_{8}^{3}$ arc kcpL. 
Based on thesc preliminary calculations, we "correct" the temporary value of $\mathrm{c}_{13}^{0}$ by a quantity $\Delta \varepsilon_{13}^{0}$ and we also consider new values $c_{15}^{0}+\Delta c_{15}^{0}$ for $c_{15}^{0}$. The variation $\Delta c_{13}^{0}$ is sclected in such a way that for any $\Delta e_{15}^{0}$ value satisfying $\Delta e_{15}^{0} \equiv 0$, the new value $e_{8}^{3}+\Delta e_{8}^{3}$ of $e_{8}^{3}$ is equal to $-2^{8}$ with a substantial probability.

$$
\text { Proposivion } 6 \text { : If } \mathrm{e}_{12}^{2} \neq 0 \text { and } \frac{-2^{8}-e_{8}^{3}}{2^{4.4 .7} \mathrm{e}_{12}^{2}} \equiv 0 \text { and } \mathrm{e}_{\mathrm{j}}^{2} \bmod 16 \text { is not in }(13,15) \text { for } 1 \leq j \leq 11 \text { then for }
$$
any variation $\Delta c_{15}^{0} \equiv 0$ on $c_{15}^{0}$ such that $c_{15}^{2}+\Delta c_{15}^{0}<p$ and $c_{15}^{4}+\Delta c_{15}^{0}<p$, the variation $\Delta e_{13}^{0}=\frac{-2^{8}-e_{8}^{3}}{4.4 .7 e_{12}^{2}}$ on the $e_{13}^{0}$ value leads to the following new valucs : $c_{14}^{1}+\Delta c_{14}^{1}=0 ; \quad c_{0}^{2}+\Delta c_{0}^{2}=0 ; \quad c_{6}^{2}+\Delta c_{6}^{2}=0 ; \quad c_{8}^{2}+\Delta c_{8}^{2}=0 ; \quad c_{8}^{3}+\Delta c_{8}^{3}=-2^{8}$.

We performed a number $n_{1}$ of trials of step l. For cach sucecssful trial of step 1, we made a large number $\mathrm{n}_{2}$ of trials of $c_{11}^{0}$ values at step 2. For those $c_{11}^{0}$ values satisfying the conditions of the above proposition, we made a large number $n_{3}$ of trials of new $c_{15}^{0}$ valucs such that $\Delta c_{15}^{0} \equiv 0$. The probability that the trial of a new $\Delta c_{15}^{0}$ value leads to intermediate variables satisfying the four cquations $c_{6}^{2}=0 ; c_{8}^{2}=0$; $e_{6}^{4}=0 ; e_{8}^{4}=0$ is basically the probability that randomiy tried $e_{6}^{4}$ and $c_{5}^{4}$ values satisfy $c_{6}^{4}=0$ and $e_{5}^{4} \equiv 6$; the order of magnitude of this probability is thercfore $2^{-20}$.

Moreover, the probability that a message $M_{2}$ satisfying the four cquations $c_{6}^{2}=0 ; c_{8}^{2}=0 ; c_{6}^{4}=0 ; c_{8}^{4}=0$ lcads to a full collision $\mathrm{g}\left(\mathrm{H}_{1}, \mathrm{M}_{2}\right)=\mathrm{g}\left(\mathrm{H}_{1}, \mathrm{M}_{2}\right)$ is basically the probability that none of the $\mathrm{c}_{\mathrm{i}-3}$ mod 16 indices occurring in the calculation of $e_{0}^{2}$ to $e_{15}^{2}$ and of $c_{0}^{4}$ to $c_{15}^{4}$ takes the value 15 , which is close to $1 / 8$. So in summary approximatively $2^{23}$ partial computations of the g function are necessary to obtain a message $\mathrm{M}_{2}$ giving a full collision. 


\subsection{Implementation details}

The above attack method was implemented using a non-optimized Pascal program. The scarch for a collision took a fcw hours on a SUN3 workstation and less than an hour on a SPARC workstacion. Wc provide in annex the detail of the intermediate calculations for two colliding messages $M_{1} M_{2}$ and $M_{1} M_{2}$. of two 128-bit blocks cach.

Note that for many other values $M^{\prime \prime}$, of the form $\left(c_{0}^{0}, \ldots, c_{15}^{0}+k\right.$.16) (k: an integer) of the first 128-bit block, the message $M^{\prime \prime}{ }_{1} M_{2}$ leads to the same hash value as $M_{1} M_{2}$ : the observed phenomenon is in fact a multiolecollision.

\section{Conclusions}

The attack described in this paper takes advantage of the two following wcaknesses of the FFTHashing algorithm :

- the influence of the term $c_{c_{i-3}}$ in the recurrence $c_{i}:=c_{i}+c_{i-1} e_{i-2}+c_{c_{i-3}}+2^{i}$ (mod $p$ ) on the security of the algorithm is rather negative (sec for example the method to obtain $c_{6}^{2}=0$ (or $c_{8}^{2}=0$ ) at step 1 of Scction 2.2).

- as mentioned in Section 1.3, the diffusion introduced by the four steps of the algorithm is quite limited. In particular, the $\mathrm{FT}_{8}$ Fourier transfiorm acts only on half of the intermediate values $\left(e_{0}, \ldots, e_{15}\right)$, namely the 8 values $e_{0}, c_{2}, \ldots, c_{14}$

This suggests that quite simple modifications might result in a substantial improvement of the security of the FFT-Hashing algorithm.

\section{Acknowledgements}

The autors are grealcful to Jacques BURGER (SEPT PEM, 42 rue des Coutures, BP 6243, 14066 CAEN, France) for the Spare implementation as well as uscful discussions.

\section{References}

[1] : C.P. SCHNORR; FFT-Hashing : An Efricicnt Cryptographic Hash Function; July 15, 1991 (This paper was prescried at the rump session of the CRYPTO'91 Conference, Santa Barbara, August, 11-15, 1991)

[2] : DAEMEN - BOSSELAERS - GOVAERTS - VANDEWALLE : Announcement made at the rump session of the ASIACRYPT '91 Conference, Fujiyoshida, Japan, November 11-14, 1991) 


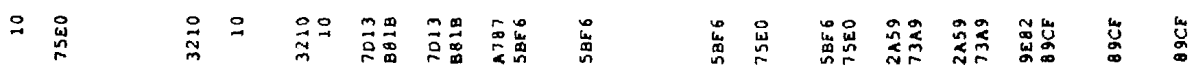

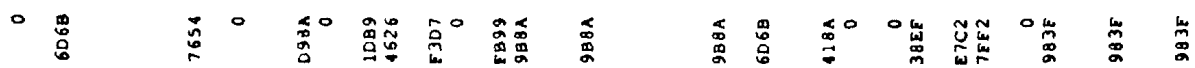

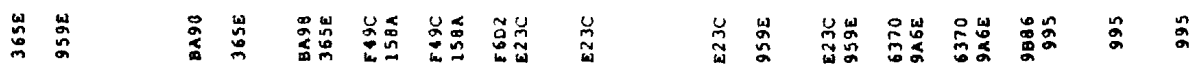

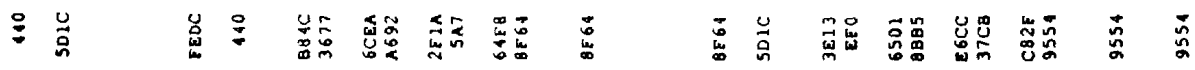

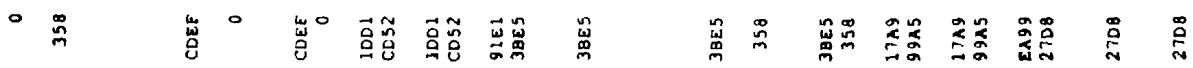

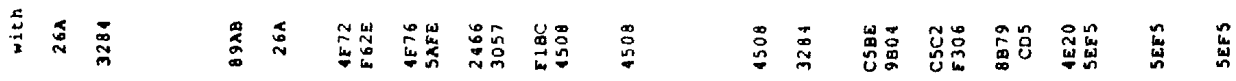

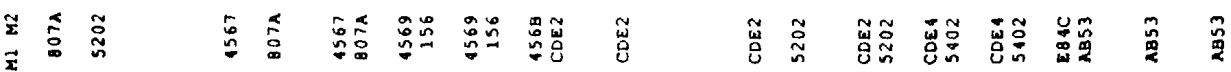

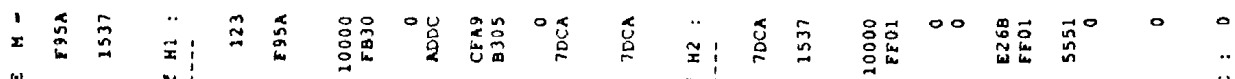

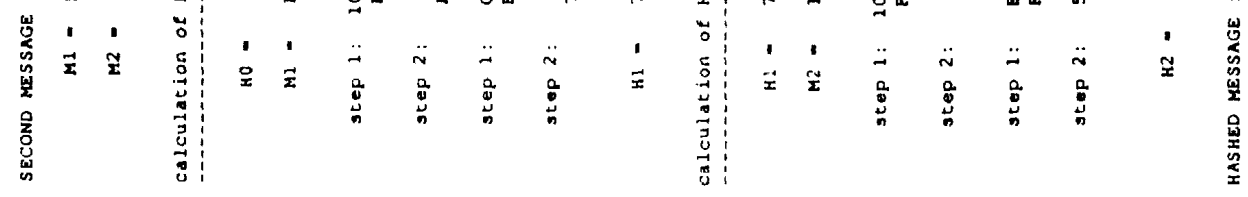

妾

•

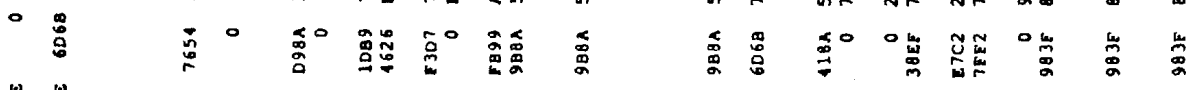

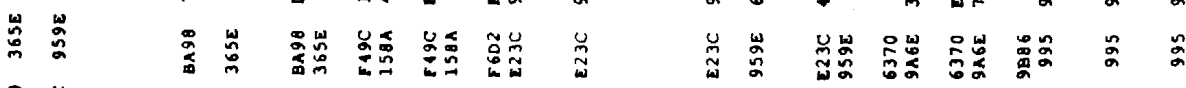

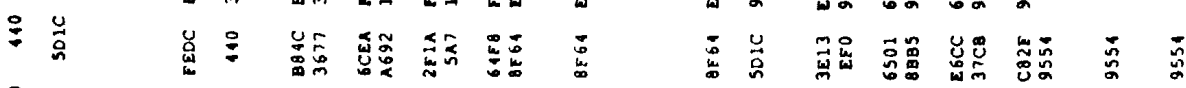

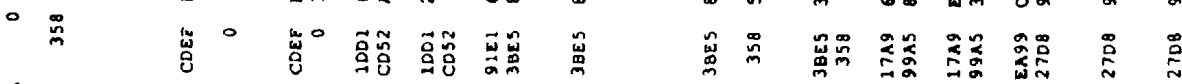

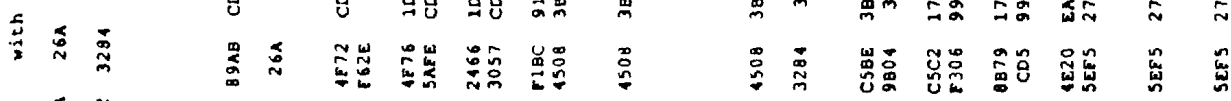

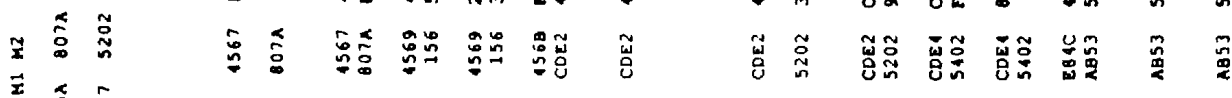

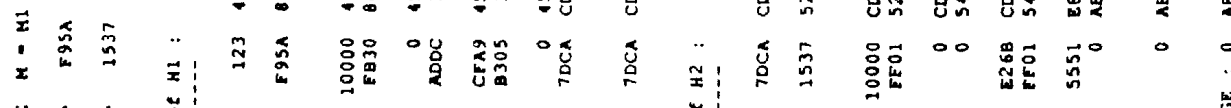

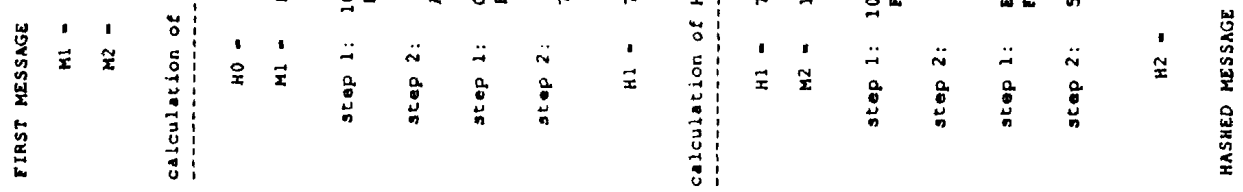

